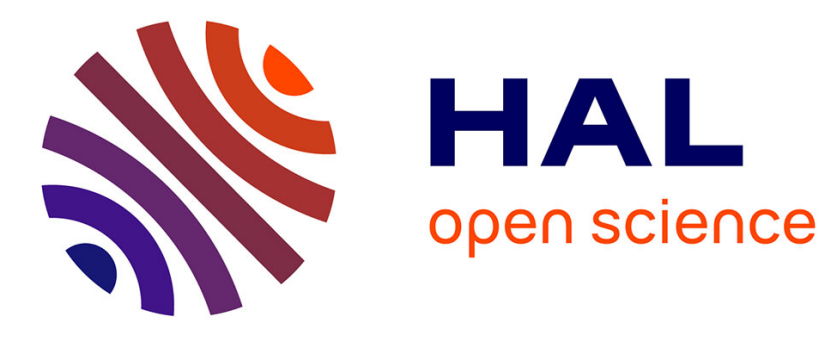

\title{
Controlling internal barrier in low loss BaTiO3 supercapacitors
}

U-Chan Chung Seu, Catherine Elissalde, Stéphane Mornet, Mario Maglione, Claude Estournès

\section{- To cite this version:}

U-Chan Chung Seu, Catherine Elissalde, Stéphane Mornet, Mario Maglione, Claude Estournès. Controlling internal barrier in low loss BaTiO3 supercapacitors. Applied Physics Letters, 2009, 94 (7), 072903 (3 p.). 10.1063/1.3076125. hal-00369121

\section{HAL Id: hal-00369121 \\ https://hal.science/hal-00369121}

Submitted on 7 Apr 2009

HAL is a multi-disciplinary open access archive for the deposit and dissemination of scientific research documents, whether they are published or not. The documents may come from teaching and research institutions in France or abroad, or from public or private research centers.
L'archive ouverte pluridisciplinaire HAL, est destinée au dépôt et à la diffusion de documents scientifiques de niveau recherche, publiés ou non, émanant des établissements d'enseignement et de recherche français ou étrangers, des laboratoires publics ou privés. 


\title{
Controlling internal barrier in low loss $\mathrm{BaTiO}_{3}$ supercapacitors
}

\author{
U-C. Chung, C. Elissalde, S. Mornet and M. Maglione \\ ICMCB - CNRS, Université Bordeaux, 87 Avenue du Dr A. Schweitzer, F-33608 Pessac \\ cedex, France
}

C. Estournès

CNRS; Institut Carnot Cirimat; F-31062 Toulouse, France et Plateforme Nationale de Frittage Flash, PNF2-CNRS, 118 route de Narbonne, 31062 Toulouse cedex 09, France

Supercapacitor behavior has been reported in a number of oxides including reduced $\mathrm{BaTiO}_{3}$ ferroelectric ceramics. These so-called giant properties are however not easily controlled. We show here that the continuous coating of individual $\mathrm{BaTiO}_{3}$ grains by a silica shell in combination with Spark Plasma Sintering is a way to process bulk composites having supercapacitor features with low dielectric losses and temperature stability. The silica shell acts both as an oxidation barrier during the processing and as a dielectric barrier in the final composite. 
Commercial supercapacitors are using polycrystalline materials in which metallic grains or foils (like tantalum) with extended specific surface are covered by their respective oxides (like tantalum oxide). The resulting barrier layers locate electronic and ionic space charges, increasing the capacitance density. While such barrier layer supercapacitors are of everyday use, ${ }^{1,2}$ an improved control of the interfaces barrier in such advanced materials would lead to increased functionalities: higher charge storage ability, lower losses and increased cut-off frequency. We show here that individual coating of ferroelectric $\mathrm{BaTiO}_{3}$ grains by a silica shell followed by a fast sintering in reducing conditions leads to such stable supercapacitor behavior in electroceramics. The tuning of a dielectric shell at the grain scale, as compared with standard routes, offers several opportunities for electroceramics.

Actually, there is an intense wave of research on materials displaying extreme dielectric parameters. Many compounds such as $\mathrm{CaCu}_{3} \mathrm{Ti}_{4} \mathrm{O}_{12}$ and $\mathrm{LuFe}_{2} \mathrm{O}_{4}$ have been quoted as "giant" permittivity materials. Well known and widely used ceramics like $\mathrm{BaTiO}_{3}$ show huge effective dielectric parameters when appropriately substituted or when decreasing the grain size under fast sintering conditions. ${ }^{3-6}$ In all cases, the apparent dielectric permittivity is high at room temperature $\left(\varepsilon>10^{5}\right.$ in some cases) and is temperature independent in a broad range before falling down at low temperatures. While these features are very appealing for applications, some parameters are still not controlled: the dielectric losses are high and low frequency contributions to the dielectric permittivity are ascribed to dc conductivity. In $\mathrm{CaCu}_{3} \mathrm{Ti}_{4} \mathrm{O}_{12}$ ceramics, this dielectric behavior was ascribed to an internal barrier layer (IBLC) at the grain boundaries. ${ }^{7}$ Within this model, the measured huge capacitances are not increased by intrinsic dielectric parameters but by geometrical confinement of the dielectric at the grain boundaries. All the above mentioned materials can be described using this IBLC model, each of them having different free charges able to increase the inner grain 
conductivity. ${ }^{8}$ However, the key parameter which is the grain boundary interface, is still not well understood and controlled. This is a strong drawback in order to reproduce the dielectric performances and to reduce the dielectric losses of IBLC ceramics. We propose here to solve this issue by using advanced processing of core@shell particles sintered using Spark Plasma Sintering (SPS). ${ }^{9,10}$ By means of homogeneously coated $\mathrm{BaTiO}_{3}$ grains with an amorphous silica shell, we were able to obtain composite ceramics of apparent permittivity of $2.10^{5}$ and losses of only a few percent at $10^{4} \mathrm{~Hz}$ which are stable against temperature.

The two main conditions for the internal barrier layer to increase the overall dielectric permittivity of oxides are the inner grain conductivity and the insulating grain boundary. The former is related to the amount of charged defects which are intentionally introduced prior to the precursor reaction or during the sintering step under reducing conditions. ${ }^{6,11}$ The stabilization of such charged lattice defects in a reproducible way in $\mathrm{BaTiO}_{3}$ ceramics, is still a challenge. Even the way to compensate for heterovalent substitution like $\mathrm{La}^{3+}$ on the $\mathrm{Ba}^{2+}$ site of $\mathrm{BaTiO}_{3}$ is still a matter of debate. ${ }^{11,12}$

The control of the grain boundaries (i.e. tuning their morphologies and electrical properties) is also not an easy task. Indeed, depending on the size and the chemical state of the starting grains, the sintering step of the bulk ceramics can strongly alter shape, charges and electronic state of the grain boundaries. For $\mathrm{BaTiO}_{3}$, many discrepancies have been quoted in the literature depending on the processing route. ${ }^{13-15}$ This is even more true when fast sintering processes under reducing atmosphere like Spark Plasma Sintering are used.

This is why we decided to coat each $\mathrm{BaTiO}_{3}$ grain prior to the sintering step in order to improve the reproducibility of the grain boundaries in dense ceramics. The first step is to produce core@shell powders made of $\mathrm{BaTiO}_{3}$ cores surrounded by a continuous and homogeneous shell of amorphous silica $\left(\mathrm{SiO}_{2}\right)$. We previously showed that the thickness of 
the shell can be tuned continuously from few nanometers up to tenths of microns. This core@shell architecture was efficient to decrease the dielectric losses of the final ceramics and also to achieve multifunctionality on including nanoparticles in the coating. ${ }^{9,16}$ For the present purpose of achieving extremely high and reproducible dielectric permittivity ceramics, we started from standard $\mathrm{BaTiO}_{3}$ particles of $500 \mathrm{~nm}$ diameter purchased from Sakai Chemical Co. (Japan). All the particles have been individually coated with a homogeneous amorphous silica shell of $5 \mathrm{~nm}$ thickness using a method derived from the Stöber process, as described in detail elsewhere. ${ }^{9}$ Core@shell $\mathrm{BaTiO}_{3}(500 \mathrm{~nm}) @ \mathrm{SiO}_{2}(5 \mathrm{~nm})$ particles are denoted as [BT500@Si5].

Once the starting core@shell particles are available, the sintering step should also be optimized since any porosity is detrimental for getting large capacitance density. Improved two-step sintering has been used but long dwelling times are required $\left(950^{\circ} \mathrm{C}, 12 \mathrm{~h}\right)$ and the resulting density does not reach $85 \% .{ }^{17}$ As to build an IBLC ceramic, we then need to induce charged point defects in the ceramics. It has been previously shown that SPS, which is operated under reducing atmosphere, is able to create such defects as $\mathrm{Ti}^{3+}$ associated to oxygen vacancies. ${ }^{6}$ The SPS experiments were performed in a Dr Sinter SPS-2080 Syntex INC Japan. A heating rate of $100^{\circ} \mathrm{C} \cdot \mathrm{min}^{-1}$ was used to reach the final temperature of $1100^{\circ} \mathrm{C}$ under vacuum. A uniaxial pressure of $50 \mathrm{MPa}$ was applied immediately before and until completion of the temperature rising step. The as obtained ceramics have a surface layer contaminated by carbon coming from the graphite die in which the pellets are pressed. A postannealing step at $800^{\circ} \mathrm{C}$ for $12 \mathrm{~h}$ in air is performed for decarbonation of the samples; the density reached $97 \%$ for all of them. After this first post-annealing treatment, the pellet remains navy blue stemming from the reduction state of titanium (this color being usually ascribed to $\mathrm{Ti}^{3+}-\mathrm{VO}$ centers). 
A second identical thermal treatment did not allow re-oxidizing the sample, highlighting the efficiency of silica as a barrier against re-oxidation. Considering the extreme SPS sintering conditions, the silica in the final pellet, can be viewed as a glassy matrix in which the BT grains are embedded as evidenced by High Resolution SEM. The grain size remains close to the initial one (i.e. $500 \mathrm{~nm}$ ) and the fused aspect on the grains surface is attributed to the glassy silica matrix (Fig.1).

Having confirmed that charged defects are indeed stable in the ceramics, we next need to check the silica shell at the grain boundaries. In a separate paper, we used in situ Transmission Electron Microscopy (TEM), High Resolution TEM and XPS, showing the preservation of the silica coating under the mentioned SPS conditions [submitted]. We point out that cross diffusion between silica and $\mathrm{BaTiO}_{3}$ is moderate and that amorphous silica is still surrounding the grains in the ceramics. The capacitance and dielectric losses of the samples were recorded as a function of temperature $(4 \mathrm{~K}-450 \mathrm{~K})$ and frequency $(100 \mathrm{~Hz}-$ $10 \mathrm{MHz}$ ). On figure 2, we report the real part of the dielectric permittivity ( $\left.\varepsilon^{\prime}\right)$ and the dielectric losses $(\operatorname{tg} \delta$ ) in the temperature range 300-450 K for several spot frequencies. These parameters are only apparent ones and even if they should be considered with some care, because of the IBLC model, we will, however, discuss them for sake of comparison with the existing literature. First, the room temperature dielectric permittivity is in the range of the so called giant dielectric materials $\left(\varepsilon \sim 2.10^{5}\right.$ at $\left.\mathrm{f}=10^{4} \mathrm{~Hz}\right)$ meaning that our core shell particles are indeed leading to IBLC ceramics (Fig. 2a). The main achievement here is that the dielectric losses are much smaller than the ones previously reported i.e. tg $\delta \sim 5 \%$ at $\mathrm{f}=10^{4} \mathrm{~Hz}$ instead of $100 \%$ in the existing literature (Fig. 2b). Moreover, the absence of apparent conductivity contribution at low frequencies shows a small conductivity across the whole ceramic even at $\mathrm{T}>100^{\circ} \mathrm{C}$. These two main features, lower dielectric losses and the absence of 
a conductivity contribution may be ascribed to the silica coating. Another interesting feature is the small maximum in the dielectric permittivity at the ferroelectric/paraelectric transition temperature $\mathrm{T}=400 \mathrm{~K}$ (Fig. 2a). This means that the bulk ferroelectric properties of the material are not totally cancelled by the IBLC effect.

To get a more quantitative insight into this IBLC contribution to the increased dielectric parameters, one should follow the dielectric relaxation which inevitably occurs for all of similar materials. ${ }^{8}$ Such relaxation clearly appears on figure 3a where the real part of the dielectric permittivity bends down sharply at low temperatures. As for every supercapacitor, the high frequency permittivity is thus much lower than the low frequency one. The relaxation is better seen on the losses which undergo a strong maximum and whose temperature is shifted with the operating frequency (Fig. 3b). Both the shape and the shift of this maximum stem from a dielectric relaxation which is nothing but the relaxation of the space charges located at the grain boundaries. The activation energy of this space charge may be mapped out on plotting the characteristic frequencies and temperatures in an Arrhenius plot (Fig. 3a inset). The linear evolution evidences the exponential behavior of the relaxation frequency $f=f_{0} \exp -\left(\frac{E}{k T}\right)$ where $\mathrm{f}_{0}$ is the high temperature extrapolation of the relaxation frequency and $\mathrm{E}$ its activation energy; $\mathrm{E}=0.15 \mathrm{eV}$ which agrees with similar relaxations observed in many other materials. ${ }^{8}$ This supports space charge relaxation at the IBLC as a probable mechanism. Indeed, $0.15 \mathrm{eV}$ is in the range of the $\mathrm{Ti}^{3+}$ level position below the conduction band of $\mathrm{BaTiO}_{3}$. Electron hopping between such defects and their localization at the interface is thus a way to artificially increase the dielectric properties. ${ }^{18,19}$

Compared with IBLC ceramics referred in literature, our core@shell composites exhibit unprecedented behavior as they combine both giant permittivity and stable dielectric 
losses as low as $5 \%$ at $10 \mathrm{kHz}$. In a similar way, our structures can be called ISBLC ( $S$ for silica). The occurrence of the Curie temperature maximum indicates that the bulk properties of the composite are not cancelled by the silica barrier layer. Microstructural investigations allowed to describe the final composite structure as ferroelectric grains embedded in a glassy matrix. SPS does not only help for densification but allows to quench the amorphous silica in a glassy state thanks to the extremely fast sintering and cooling rates. In turn, this glassy silica cage allows an irreversible reduction of the BT core (induced by the reducing SPS sintering conditions). As a result, the SPS allows acting on both the core and the shell. The silica is both a dielectric barrier and a re-oxidation barrier leading to low loss supercapacitor. In addition, its thickness can be easily controlled leading to reproducibility and reliability of our composite properties. The supercapacitor behavior has been ascribed to space charge relaxation associated to $\mathrm{Ti}^{4+}$ reduction.

This work was supported by the French National Agency for Research under contract ANR NANO4F. 


\section{REFERENCES}

${ }^{1}$ D. S. Campbell, Radio Electron. Engn. 41, 5 (1971).

${ }^{2}$ J. M. Albella, I. Montero, J. M. Martinez-Duart, V. Parkhutik, Journal of Materials Science 26, 3422 (1991).

${ }^{3}$ A. P. Ramirez, M. A. Subramanian, M. Gardel, G. Blumberg, D. Li, T. Vogt and S. M. Shapiro, Solid State Com. 115, 217 (2000).

${ }^{4}$ N. Ikeda, H. Ohsumi, K. Ohwada, K. Ishii, T. Inami, K. Kakurai, Y. Murakami, K. Yoshii, S. Mori, Y. Horibe and H. Kito, Nature 436, 1136 (2005).

${ }^{5}$ I. P. Raevski, S. A. Prosandeev, A. S. Bogatin, M. A. Malitskaya and L. Jastrabik, J. Appl. Phys. 93, 4130 (2003).

${ }^{6}$ S. Guillemet-Fritsch, Z. Valdez-Nava, C. Tenailleau, T. Lebey, B. Durand and J. Y. ChaneChing, Adv. Mater. 20, 551 (2008).

${ }^{7}$ D. C. Sinclair, T. B. Adams, F. D. Morrison, and A. R. West, Appl. Phys. Lett. 80, 25 (2002).

${ }^{8}$ M. Maglione, "Charge Transfer and Vibronic States in Ionic-Covalent Systems" Theory, Experiment, and Applications, edited by V.S. Vikhnin and G.K. Liu,Springer/Tsinghua University Press, Springer Series of Topics in Solid-State Sciences, in press (2008). 
${ }^{9}$ S. Mornet, C. Elissalde, V. Hornebecq, O. Bidault, E. Duguet, A. Brisson, and M. Maglione, Chem. Mater. 17, 4530 (2005).

${ }^{10}$ U-C. Chung, C. Elissalde, M. Maglione, C. Estournès, M. Paté and J. P. Ganne, Appl. Phys. Lett. 92, 042902 (2008).

${ }^{11}$ D. M. Smyth, J. of Electroceramics 9, 179 (2002).

${ }^{12}$ F. D. Morrison, A. M. Coats, D. C. Sinclair and A. R. West, J. of Electroceramics 6, 219 (2001).

${ }^{13}$ X. Wang, X. Deng, H. Wen and L. Li, Appl. Phys. Lett. 89, 162902.1 (2006).

${ }^{14}$ M. T. Buscaglia, M. Viviani, V. Buscaglia, L. Mitoseriu, A. Testino, P. Nanni, Z. Zhao, M. Nygren, C. Harnaga, D. Piazza and C. Galassi, Phys. Rev. B 73, 064114-1 (2006).

${ }^{15}$ D. H. Yoon and B. I. Lee, J. of Ceramic Processing Research 3, 41 (2002).

${ }^{16}$ S. Mornet, C. Elissalde, O. Bidault, F. Weill, E. Sellier, O. Nguyen and M Maglione, Chem. Mater. 19, 987 (2007).

${ }^{17}$ I. W. Chen and X. H.Wang, Nature 404, 168 (2000). 
${ }^{18}$ O. Bidault, P. Goux, M. Kchikech, M. Belkaoumi and M. Maglione, Phys. Rev. B 49, 7868 (1994).

${ }^{19}$ O. Bidault, M. Actis and M. Maglione, Solid State Comm. 95, 845 (1995). 


\section{FIGURE CAPTIONS}

FIG. 1. HRSEM micrograph of the fracture of $\mathrm{BaTiO}_{3}(500 \mathrm{~nm}) @ \mathrm{SiO}_{2}(5 \mathrm{~nm})$ sintered by SPS at $1100^{\circ} \mathrm{C}$ under vacuum.

FIG. 2. (a) Thermal evolution of the real part of the permittivity at $10 \mathrm{kHz}$ after a first and a second post-annealing. (b) Thermal evolution of loss tangent at $10 \mathrm{kHz}$ after a first and a second post-annealing.

FIG. 3. (a) Dielectric permittivity at low temperature $(\mathrm{T}<300 \mathrm{~K})$ for several frequencies in the range $100 \mathrm{~Hz}-1 \mathrm{MHz}$. (Inset) The activation energy of this relaxation is $0.15 \mathrm{eV}$ which is consistent with what reported in many different materials. (b) Dielectric losses at low temperature ( $\mathrm{T}<300 \mathrm{~K}$ ) for several frequencies in the range $100 \mathrm{~Hz}-1 \mathrm{MHz}$. The shift of the loss maximum stems from a dielectric relaxation whose Arrhenius behaviour is evidenced. 


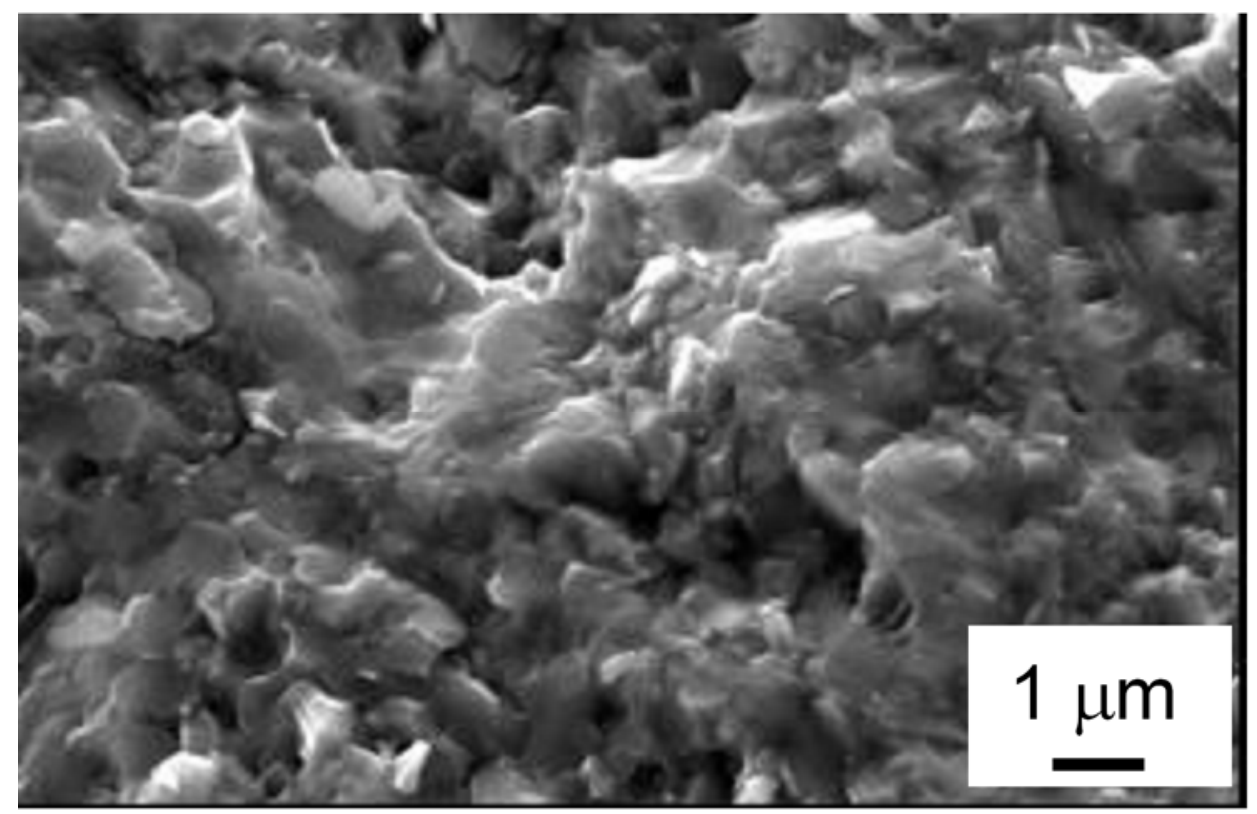

Fig. 1
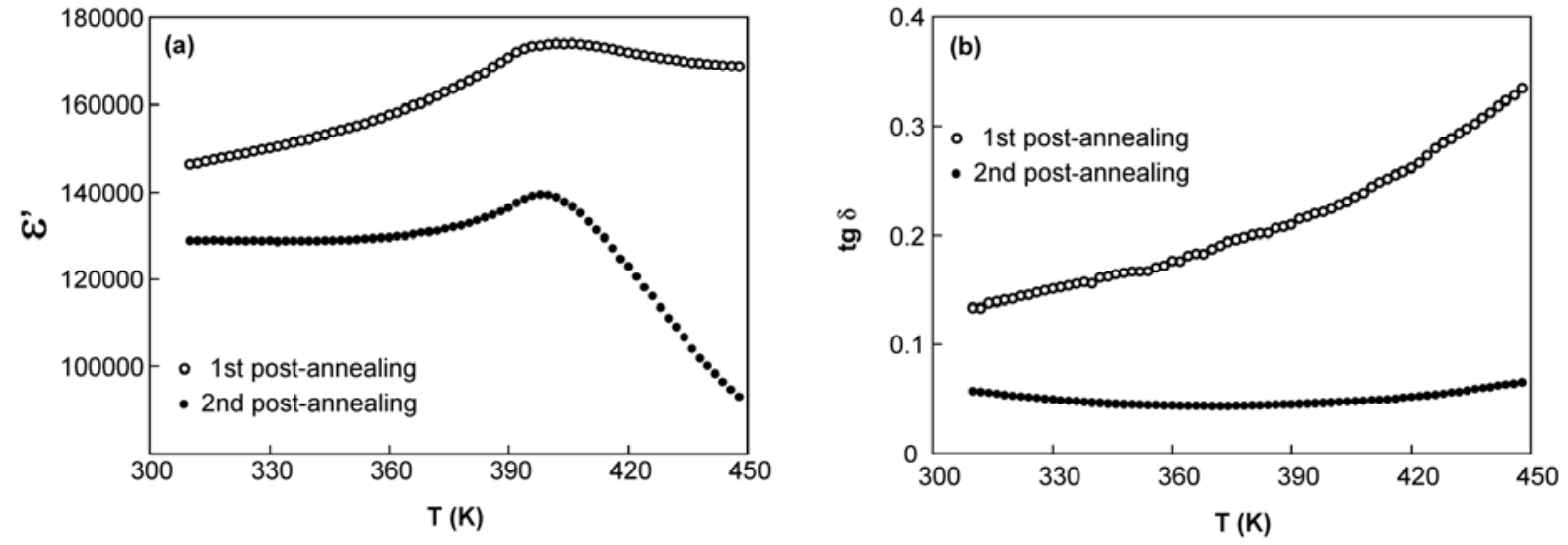

Fig. 2
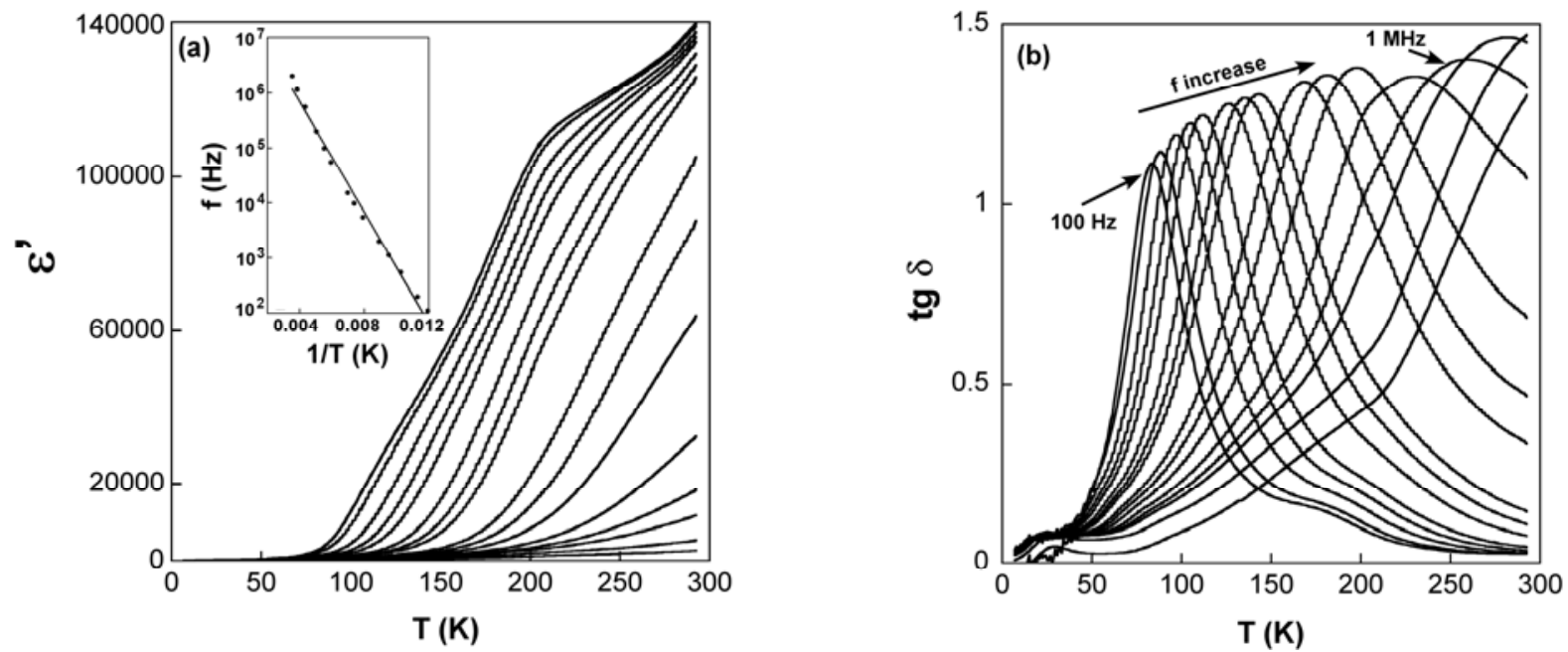

Fig. 3 